\title{
Prenatal Origins of Carpal Fusions
}

STANLEY M. GARN, ALPHONSE R. BURDI AND WILLIAM J. BABLER The Center for Human Growth and Development, and Department of Anatomy, The University of Michigan, Ann Arbor, Michigan 48109

\author{
KEY WORDS Bone · Carpals · Fusions · Metacarpal-carpal \\ Fusions.
}

\begin{abstract}
As shown in 138 embryos and fetuses in the $40-285 \mathrm{~mm}$ crownrump length range, carpal and carpal-metacarpal "fusions" arise from incomplete separation of the cartilaginous precursors rather than from failure of initiation, thus accounting for the "fusions" seen in postnatal radiographs and the grooves that are evident enough in adult fusions. Radiographs selected from over 20,000 apparently normal individuals provide postnatal counterparts for the prenatal examples shown in histological sections.
\end{abstract}

Carpal "fusions," involving pairs of carpal bones or carpals and adjacent metacarpals are familiar both to radiologists and physical anthropologists concerned with the development of the hand skeleton (Garn, '70; Poznanski, '72). While some fusions actually arise during postnatal life as the result of trauma or joint disease, the majority of fusions seen in hand radiographs of children and young adults are of prenatal origin. What appears to be a "fusion" is in actuality a failure to separate during the embryonic period (c.f. O'Rahilly, '57), due to failure of cellular death.

There are, moreover, marked population differences in the frequency of carpal fusions, especially those involving the triquetral and lunate. The os lunatotriquetrum is especially common in African children, as seen in $\mathrm{x}$-rays (Smitham, '48; MacKay, '52; Silverman, '55; Cockshott, '63) with a frequency of $8-9 \%$ in the Hausa of Nigeria. The lunate-triquetral fusion is also common in American children of (largely) West African ancestry (Garn et al., '71), as confirmed in over 20,000 hand radiographs.

So far, however, no comprehensive survey has been made of carpal and carpalmetacarpal fusions during the embryonic and fetal periods in any single embryological collection. Moreover, it has not been clear whether "fusions" arise because of complete failure to separate, because of partial failure of separation, or even be- cause of breakdown of one or more joints in embryogenesis. These questions have led us to make use of stained histological hand sections from the Patten Embryology Research Collection of the University of Michigan, including specimens added through 1974. As in our previous studies, only specimens categorized by race and accompanied by both medical histories and fetal dimensions were considered. Only those specimens shown to be free from pathology by both gross and microscopic examination were termed "normal," thereby excluding ectopic (extrauterine) implantations from that category.

Thus, restricted to race-defined and dimension-defined specimens, the present study involves hand sections of 138 human embryos, 117 of them of European ancestry (i.e., designated as "white" on the hospital records), and 21 of largely African ancestry, as indicated on the hospital records. Ninety-six of the total were considered normal, by all the criteria given above, while 42 were regarded as abnormal with respect to history, gross or microscopic appearance or implantation.

Out of the total of 138 embryos and fetuses in the $40-285 \mathrm{~mm}$ crown-rump length range considered, five $(3.6 \%)$ were characterized by incomplete cellular separation between carpals or carpals and metacarpals, well beyond the age at which such separation is ordinarily complete (table 1 ). In four of these examples, only a single pair of bones was involved, carpal and car- 
TABLF 1

Prenatal carpophalangeal "fusions" in 138 specimens

\begin{tabular}{|c|c|c|c|c|c|}
\hline $\begin{array}{l}\text { Embryo } \\
\text { number }\end{array}$ & $\begin{array}{c}\text { Crown-rump } \\
\text { length }\end{array}$ & Sex & Race & Fusions & $\begin{array}{l}\text { Critical } \\
\text { length }\end{array}$ \\
\hline & $m m$ & & & & $m m$ \\
\hline 18 & 45 & $\mathrm{~F}$ & White & Capitate-Metacarpal III & 30 \\
\hline 49 & 47 & $\mathbf{F}$ & White & Capitate-Metacarpal III & 30 \\
\hline 1432 & 54 & M & White & Lunate-Triquetral & 35 \\
\hline 1185 & 57 & M & Black & Lunate-Triquetral & 35 \\
\hline \multirow{9}{*}{1474} & 185 & $\mathbf{F}$ & Black & Capitate-Lunate & 35 \\
\hline & & & & Capitate-Hamate & 30 \\
\hline & & & & Capitate-Scaphoid & 31 \\
\hline & & & & Capitate-Trapezoid & 33 \\
\hline & & & & Trapezoid-Scaphoid & 31 \\
\hline & & & & Trapezoid-Trapezius & 30 \\
\hline & & & & Trapezium-Metacarpal I & 33 \\
\hline & & & & Hamate-Metacarpal V & 30 \\
\hline & & & & Triquetral-Pisiform & 40 \\
\hline
\end{tabular}

1 Latest age beyond which separation is ordinarily complete, but without clearly defined joint spact, as determined in the present study.

pal or carpal and metacarpal. However, one specimen (No. 1474), apparently normal by all other criteria, was characterized by a total of seven fusions involving nine bones, eight carpals and one metacarpal.

Histological hand sections of these five specimens are pictured in figure 1 . In the upper left (A) a capitate-metacarpal fusion is depicted in a longitudinal section, and in the upper right (B) a capitate-metacarpal fusion is shown in oblique section. These sections correspond to specimens No, 18 and No. 49 in table 1, and show that partial separation has taken place. The middle pair of photomicrographs (C and $D$ in fig. 1), depict two lunatotriquetral "fusions," or to be more correct partial separations, well beyond the usual age at which separation is ordinarily complete. The illustration at the lower left (E) of figure 1 portrays the older specimen with multiple carpal-to-carpal and carpal-metacarpal "fusions" (No. 1474) including the pisiform-triquetral fusion, and inclusion of the first metacarpal. The seven fusions involving nine bones are diagrammed at $F$ (lower right) using a stylized drawing adapted from O'Rahilly.

For comparison with these five histological hand sections, four "fusions" are also pictured in radiographic projections, as selected from over 20,000 postero-anterior radiographs obtained in the Ten-State $\mathrm{Nu}$ trition Survey of 1968-1970, and derived from apparently-normal subjects (fig. 2).
These four fusions include a typical capitate-hamate fusion at $\mathrm{A}$ (upper left), the most common fusion observed in subjects of European derivation. A lunate-triquetral fusion is depicted at B (upper right), appropriately enough in a subject of largelyAfrican ancestry. Illustration $\mathrm{C}$ in the lower left of figure 2 shows a trapezoid-trapezium fusion while D (lower right) demonstrates a rare trapezoid-capitate fusion. All of these fusions, shown in postnatal radiographs, have their prenatal parallels in figure 1. The radiographic projections also document the problem of confirming apparent fusions in the single postero-anterior projection, without the benefit of additional oblique radiographs.

For those concerned with carpal and carpal-metacarpal fusions, the histological sections pictured in the first figure provide a wealth of information. They suggest that the fusions later seen in radiographic projections are due not to complete failure of separation, but rather to incomplete separation, long beyond the stage or crownrump length at which the carpals are completely separated and joint spaces fully delineated. These histological sections also provide some understanding of the "grooves," evident in radiographs showing "fusion," and that may be seen in suitable anatomical preparations.

Now with only 138 embryos and fetuses, however carefully studied, we do not mean to provide a close estimate of the frequency 


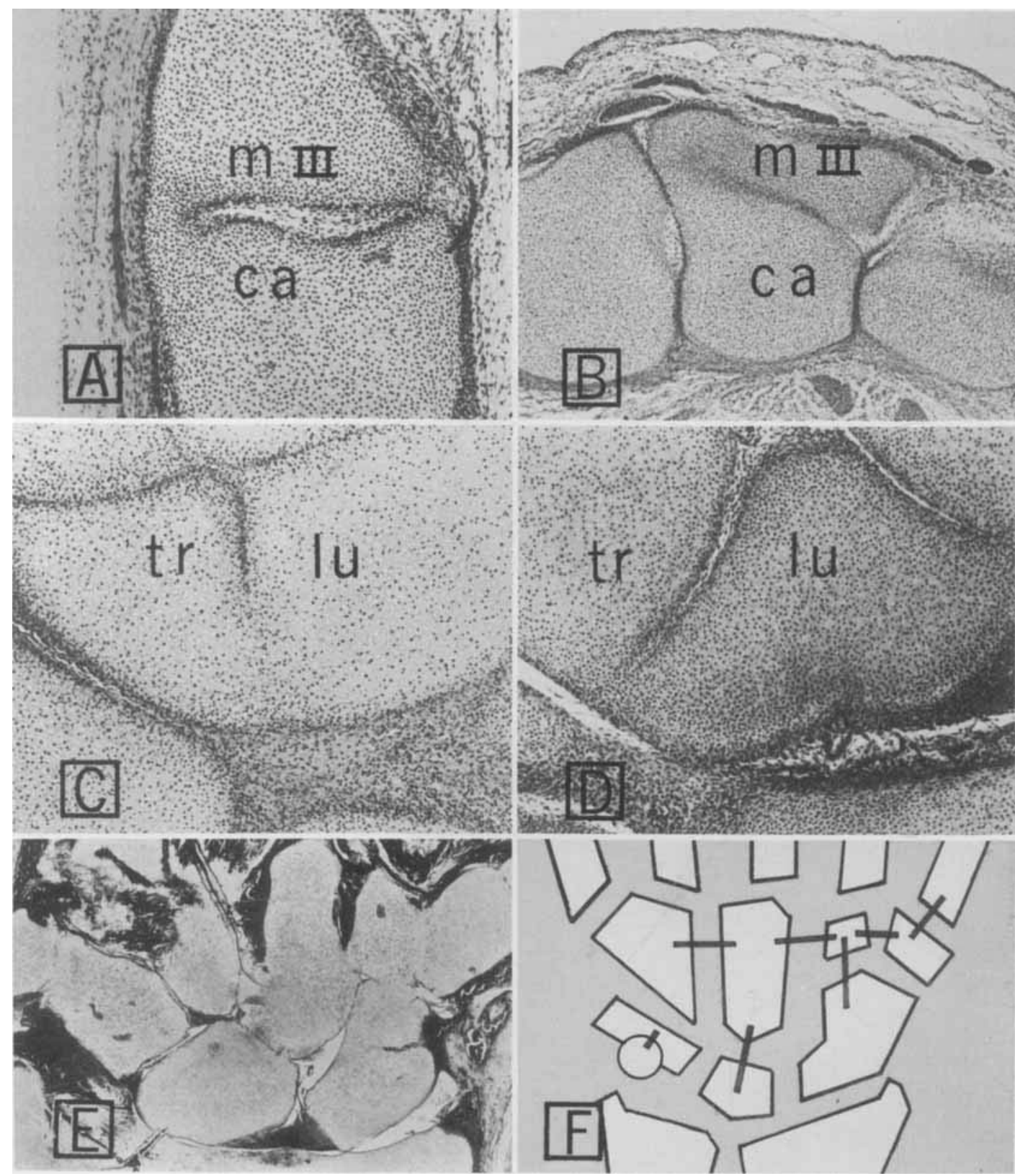

Fig. 1 "Carpal fusions" in five embryos and fetuses ranging from $45-185 \mathrm{~mm}$ in crown rump length. (A) Capitate-Metacarpal III fusion shown at $45 \mathrm{~mm}$ CRL. (B) Capitate-Metacar pal III fusion shown at $47 \mathrm{~mm}$ CRL. (C) and (D) the Lunate-Triquetral fusion at 54 and $57 \mathrm{~mm}$ CRL, respectively, showing partial separation of this pair of bones in each case. (E) Multiple fusions involving ail eight carpal bones (including the pisiform) and Metacarpal I at $185 \mathrm{~mm}$ CRL. All seven fusions in (E) are diagrammed at the lower right ( $F$ ). 


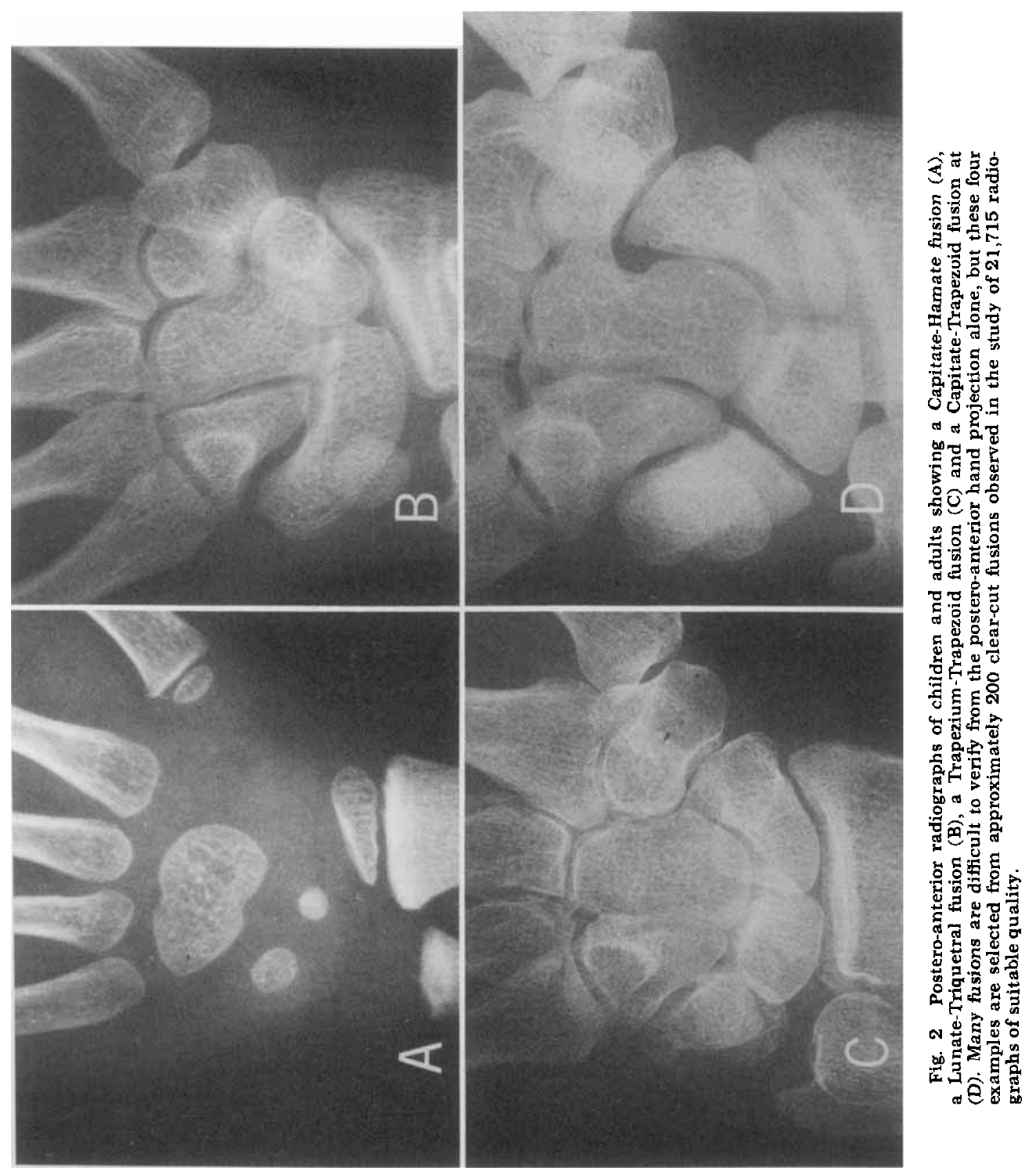


of fusions in the prenatal period, though it may be taken as $4 \%$ in the material under consideration. We would raise questions as to the "normality" of specimen 1474 with seven fusions involving nine carpal and metacarpal bones, though it is otherwise "normal" by the most stringent of anatomical criteria. It may be of interest to note that we have had to develop a whole new listing of "critical lengths," beyond which separation of carpals and carpals from metacarpals is ordinarily complete in order to provide the necessary criteria for delayed separation upon which this study largely depends.

However, it would appear that fusions (as seen in postnatal radiographs) do have their prenatal origins, not as complete failures to separate during skeletogenesis, but as partial failure of separation. It might be suggested that if initiation of cellular death (which makes for separation) is delayed, then cellular death and therefore joint separation may not proceed through to completion. Given this explanation, the high frequency of the lunate-triquetral fusion in West Africans, and in Americans of West African ancestry, may be a reflection of prenatal developmental delay, in the initiation and therefore in the completion of cellular death. Indeed, the findings in this study showing that carpal "fusions" are due to failure of complete joint separation may well explain McCredie's (75) findings that teratogens may be responsible for carpal fusions in experimental animals. Under these circumstances the most important contribution from the present study is that teratogens (as well as genes) may bring about developmental ab- normalities by preventing programmed cellular death. Again recalling the high frequency of the lunate-triquetral fusion in West Africa, it is of further interest to ascertain whether other aspects of segmentation are also involved.

\section{ACKNOWLEDGMENTS}

We wish to thank Paul Schelble for histological and photomicrographic preparations, and Dixie L. Farquharson for assistance in the manuscript completion. This research was supported in part by Grant DE 03043 from the National Institutes of Health and from the Beatrice Stanton Endowment of The University of Michigan Medical School.

\section{LITERATURE CITED}

Cockshott, W. P. 1963 Carpal fusions. Am. J Roent., 89: 1260-1271.

Garn, S. M. 1970 Human Races. Third ed. C. C Thomas, Springfield, Illinois.

Garn, S. M., A. R. Frisancho, A. K. Poznanski, J. Schweitzer and M. B. McCann 1971 Analysis of triquetral-lunate fusion. Am. J. Phys. Anthrop., 34: $431-433$

MacKay, D. N. 1952 Skeletal maturation in the hand: a study of development in East African children. Trans. Roy. Soc. Trop. Med. Hygiene, $46: 135-150$.

McCredie, J. 1975 Congenital fusion of bones: radiology, embryology and pathogenesis. Clin Radiol., 26: 47-51.

O'Rahilly, R. 1957 Developmental deviation in the carpus and the tarsus. Clin. Orthopaed, 10 : 9-18.

Poznanski, A. K. 1972 The Hand in Radiologic Diagnosis. W. B. Saunders Co., Philadelphia.

Silverman, F. N. 1955 A note on the os lunatotriquetrum. Am. J. Phys, Anthrop, 13: 143-146.

Smitham, J. N. 1948 Some observations on certain congenital abnormalities of the hand in African natives. Brit. J. Radiol., 21 : 513-518. 